\title{
Conformal (In)Equality
}

\author{
Young-Hwan Hyun ${ }^{1, \star}$, Yoonbai Kim ${ }^{1, \star \star}$, and Seokcheon Lee Le $^{2, \star \star \star}$ \\ ${ }^{1}$ Department of Physics and Institute of Basic Science, Sungkyunkwan University, Suwon 16419, Republic \\ of Korea \\ ${ }^{2}$ The Research Institute of Natural Science, Gyeongsang National University, Jinju 52828, Republic of Korea
}

\begin{abstract}
The current accelerating expansion of the Universe is explained either by dark energy or by modified gravity theories. Both of them can explain exactly the same background evolution of the Universe, however this degeneracy may be broken when the observation of large scale structure formation is taken into account. Two observables are parameterized by the so-called dark energy equation of state, $\omega$ and the growth index parameter, $\gamma$. From these observed parameters, one may reconstruct the model parameters of the so-called scalar-tensor gravity theory, one of the modified gravity theories. Especially, the scalar-tensor gravity theory is described both in Jordan frame and in Einstein frame. If cosmological observations are interpreted in one frame, then all of the observables should also be interpreted in that frame. This explicitly shows conformal inequality of cosmological observables.
\end{abstract}

\section{Introduction}

Cosmological observations support the current Universe in the phase of accelerating expansion. In the theory side, two descriptions compete. One is to adopt general relativity as the correct gravity theory and introduce the dark energy (DE) in addition to the known matter components. The other is to invoke modified gravity theories (MG) and tries to replace the DE with the modifications of the Friedmann equations as an ejective dark energy.

In this manuscript, we shall deal with one of the simplest but long-standing MG, the so-called scalar-tensor gravity (STG) which is natural in the viewpoint of string theory. When the evolution of the Universe is depicted in the context of STG, two model functions are introduced. Comparison to the observable quantities determines these two theoretically introduced functions which specifically are reconstructed in terms of the DE equation of state (EOS), $\omega[1,2]$ and the growth index parameter (GIP), $\gamma[3]$.

Once STG is considered, the question on the frame-dependence should be addressed. Since no conformal symmetry is possessed in STG coupled to matter fields even at the classical level, every conformal transformation from a chosen frame defines a new frame, which results in the set of infinitely-many physically-distinctive frames. Theoretically favored frames are two extremes, i.e., the Einstein frame (EF) and the Jordan frame (JF). The question on the frame that "where we live (now)"

\footnotetext{
^e-mail: younghwan.hyun@gmail.com

$\star \star$ e-mail: yoonbai@skku.edu

$\star \star \star$ e-mail: skylee2@gmail.com
} 
has been a long-standing debate, it seems beyond our scope at the present stage. Instead, we explicitly show that some cosmological observables are frame-dependent and if one obtains model parameters in one frame, then one should interpret all the other observables in that frame.

For this purpose, we express two functions of STG in both the JF and the EF in terms of frame-independent observable parameters, the redshift $z$ in the next section. We show the physical (in)equalities of cosmological observables in two frames such as the Hubble parameter, $H$ and the effective DE EOS, $\omega$. Thus, we show explicitly that one should keep using the only one frame for probing cosmological observables.

\section{Reconstruction of Model Functions}

We begin this section with the action of the STG in the JF [4]

$$
S=\frac{1}{16 \pi G_{*}} \int d^{4} x \sqrt{-\bar{g}}\left[F(\phi) \bar{R}-\nabla^{\mu} \phi \nabla_{\mu} \phi-2 U(\phi)\right]+S_{\mathrm{m}}\left(\bar{g}_{\mu \nu}, \psi_{\mathrm{m}}\right),
$$

where $G_{*}$ is the bare gravitational coupling constant different from the Newton's constant, $\bar{g}_{\mu \nu}$ is the JF metric, $F(\phi)$ is dimensionless function of scalar field $\phi, U(\phi)$ is its potential, and $S_{\mathrm{m}}\left(\bar{g}_{\mu \nu}\right)$ is the matter action for $\psi_{\mathrm{m}}$ representing any matter field. $F(\phi)$ is required to be positive definite to ensure attractive nature of gravity. However its specific functional form and that of $U(\phi)$ are not determined yet but should be restricted by observations. In this manuscript, all the physical variables in the JF are denoted by bars on top of them. We summarize the Friedmann equations in both frames in table 1 . In this table $\bar{H} \equiv d \ln \bar{a} / d \bar{t}$ means the Hubble parameter in the $\mathrm{JF}, \bar{\rho}_{\mathrm{m}}$ and $\bar{P}_{\mathrm{m}}$ define the matter energy density and the pressure, respectively. They are conserved quantities by the Bianchi identity. We also introduce the so-called mis-interpreted frame where the Hubble parameter is defined by $H=d \ln a / d t$ which is not the observable quantity. Some of literatures use this quantity to interpret the Hubble parameter in the EF and this is mis-interpreted [5].

\begin{tabular}{cc}
\hline \hline the frist Friedmann Eq & the second Friedmann Eq \\
\hline JF $\quad 3 F \bar{H}^{2}=8 \pi G_{*} \bar{\rho}_{\mathrm{m}}+\frac{1}{2} \bar{H}^{2} \phi^{\prime 2}-3 \bar{H}^{2} F^{\prime}+U$ & $-2 F \bar{H} \bar{H}^{\prime}=8 \pi G_{*}\left(\bar{\rho}_{\mathrm{m}}+\bar{P}_{\mathrm{m}}\right)+\bar{H}^{2} \phi^{\prime 2}+\overline{H H}^{\prime} F^{\prime}+\bar{H}^{2} F^{\prime \prime}-\bar{H}^{2} F^{\prime}$ \\
$\mathrm{EF} \quad 3 H_{\mathrm{E}}^{2}=8 \pi \frac{G_{*}}{F^{2}} \bar{\rho}_{\mathrm{m}}+\left[\frac{1}{2}\left(\frac{\phi^{\prime}}{\sqrt{F}}\right)^{2}-3 \frac{F^{\prime}}{F}+\frac{U}{F \bar{H}^{2}}\right] \frac{\bar{H}^{2}}{F}$ & $-2 H_{\mathrm{E}} \frac{d H E}{d \ln a}=8 \pi \frac{G_{*}}{F^{2}}\left(\bar{\rho}_{\mathrm{m}}+\bar{P}_{\mathrm{m}}\right)+\left[\frac{1}{2}\left(\frac{\phi^{\prime}}{\sqrt{F}}\right)^{2}+\frac{1}{2}\left(\frac{F^{\prime}}{F}\right)^{2}+\frac{F^{\prime \prime}}{F}\right] \frac{\bar{H}^{2}}{F}$ \\
$\mathrm{MF} \quad 3 H^{2}=8 \pi \frac{G_{*}}{F^{2}} \bar{\rho}_{m}+\frac{1}{F}\left[\frac{1}{2}\left(\frac{\phi^{\prime}}{\sqrt{F}}\right)^{2}+\frac{3}{4}\left(\frac{F^{\prime}}{F}\right)^{2}\right] \bar{H}^{2}+\frac{U}{F^{2}}$ & $-2 \frac{d H}{d t}=8 \pi \frac{G_{*}}{F^{2}}\left(\bar{\rho}_{\mathrm{m}}+\bar{P}_{\mathrm{m}}\right)+\frac{2}{F}\left[\frac{1}{2}\left(\frac{\phi^{\prime}}{\sqrt{F}}\right)^{2}+\frac{3}{4}\left(\frac{F^{\prime}}{F}\right)^{2}\right] \bar{H}^{2}$
\end{tabular}

Table 1. Friedmann equations in JF, EF, and mis-interpreted frame (MF). Primes mean the differentiation with respect to $\ln \bar{a}$. 
The evolution equations for the background and the matter perturbation in the flat FriedmannLemaitre-Robertson-Walker (FLRW) metric are given by [6]

$$
\begin{aligned}
& \Omega_{\mathrm{m}}(\bar{z})=\frac{\Omega_{\mathrm{m} 0}}{\Omega_{\mathrm{m} 0}+\left(1-\Omega_{\mathrm{m} 0}\right)(1+\bar{z})^{3\left(\omega_{0}+\omega_{a}\right)} e^{-3 \omega_{a} \bar{z} /(1+\bar{z})}} \\
& \frac{\bar{H}^{2}(\bar{z})}{\bar{H}_{0}^{2}}=\Omega_{\mathrm{m} 0}(1+\bar{z})^{3}+\left(1-\Omega_{\mathrm{m} 0}\right)(1+\bar{z})^{3\left(1+\omega_{0}+\omega_{a}\right)} e^{-3 \omega_{a} \bar{z} /(1+\bar{z})}, \\
& \frac{\bar{H}^{\prime}(\bar{z})}{\bar{H}(\bar{z})}=-(1+\bar{z}) \frac{\bar{H}_{, \bar{z}}}{\bar{H}}=-\frac{3}{2}\left[1+\omega(\bar{z}) \frac{\left(1-\Omega_{\mathrm{m} 0}\right)(1+\bar{z})^{3\left(\omega_{0}+\omega_{a}\right)} e^{-3 \omega_{a} \bar{z} /(1+\bar{z})}}{\Omega_{\mathrm{m} 0}+\left(1-\Omega_{\mathrm{m} 0}\right)(1+\bar{z})^{3\left(\omega_{0}+\omega_{a}\right)} e^{-3 \omega_{a} \bar{z} /(1+\bar{z})}}\right], \\
& \bar{\omega}(\bar{z})=-1+2(1+\bar{z}) \frac{(1+\bar{z}) \frac{\phi_{\bar{z}}^{2}}{F_{0}}+(1+\bar{z}) \frac{F_{\bar{z} \bar{z}}}{F_{0}}+\left(2+(1+\bar{z}) \frac{\bar{H}_{\bar{z}}}{\overline{\bar{H}}}\right) \frac{F_{\bar{z}}}{F_{0}}-2\left(\frac{F}{F_{0}}-1\right) \frac{\bar{H}_{\bar{z}}}{\overline{\bar{H}}}}{(1+\bar{z})^{2} \frac{\phi_{\bar{z}}^{2}}{F_{0}}+6(1+\bar{z}) \frac{F_{\bar{z}}}{F_{0}}-6\left(\frac{F}{F_{0}}-1\right)+\frac{2 U}{F_{0} \bar{H}_{0}^{2}} \frac{\bar{H}_{0}^{2}}{\bar{H}^{2}}} \\
& \frac{\phi_{, \bar{z}}}{\sqrt{F_{0}}}=-\sqrt{-\frac{F_{, \bar{z} \bar{z}}}{F_{0}}-\frac{1}{(1+\bar{z})}\left(2+(1+\bar{z}) \frac{\bar{H}_{, \bar{z}}}{\bar{H}}\right) \frac{F_{, \bar{z}}}{F_{0}}+\frac{2}{(1+\bar{z})} \frac{\bar{H}_{\bar{z}}}{\bar{H}} \frac{F}{F_{0}}-3 \frac{\Omega_{\mathrm{m}}(\bar{z})}{(1+\bar{z})^{2}}} \\
& \frac{F_{, \bar{z}}}{F_{0}}=\left(\frac{3}{(1+\bar{z})}-2 \frac{\bar{H}_{, \bar{z}}}{\bar{H}}-\frac{P_{, \bar{z}}}{P}\right) \frac{F}{F_{0}}, \\
& \frac{F_{, \bar{z} \bar{z}}}{F_{0}}=\left(\left[\frac{3}{(1+\bar{z})}-2 \frac{\bar{H}_{, \bar{z}}}{\bar{H}}-\frac{P_{, \bar{z}}}{P}\right]^{2}-\frac{3}{(1+\bar{z})^{2}}-2\left[\frac{\bar{H}_{, \bar{z}}}{\bar{H}}\right]_{, \bar{z}}-\left[\frac{P_{, \bar{z}}}{P}\right]_{, \bar{z}}\right) \frac{F}{F_{0}},
\end{aligned}
$$

where $\bar{H}_{0} \equiv \bar{H}(\bar{z}=0)$, subscript $z$ means the differentiation with respect to redshift which related to $F_{, \bar{z}}=-F^{\prime} /(1+\bar{z})$ and DE EOS is parametrized as the so-called CPL parametrization

$$
\omega(\bar{z})=\omega_{0}+\omega_{a} \frac{\bar{z}}{1+\bar{z}} .
$$

$F / F_{0}$ is given by

$$
\begin{aligned}
\frac{F(\bar{z})}{F_{0}}= & \frac{3}{2} \frac{\Omega_{\mathrm{m}}(\bar{z})}{P(\bar{z})}, \\
P(\bar{z})= & \Omega_{\mathrm{m}}(\bar{z})^{\gamma}\left(\Omega_{\mathrm{m}}(\bar{z})^{\gamma}-(1+\bar{z}) \gamma_{, \bar{z}} \ln \Omega_{\mathrm{m}}(\bar{z})-\gamma\left[3-2(1+\bar{z}) \frac{\bar{H}_{, \bar{z}}}{\bar{H}}\right]+2-(1+\bar{z}) \frac{\bar{H}_{, \bar{z}}}{\bar{H}}\right), \\
\frac{U(\bar{z})}{F_{0} \bar{H}_{0}^{2}=} & \frac{1}{2} \frac{\bar{H}^{2}}{\bar{H}_{0}^{2}}\left((1+\bar{z})^{2} \frac{F_{, \bar{z}}}{F_{0}}+(1+\bar{z}) \frac{F_{, \bar{z}}}{F_{0}}-(1+\bar{z})\left[5-(1+\bar{z}) \frac{\bar{H}_{, \bar{z}}}{\bar{H}}\right] \frac{F_{, \bar{z}}}{F_{0}}\right. \\
& \left.+2\left[3-(1+\bar{z}) \frac{\bar{H}_{, \bar{z}}}{\bar{H}}\right] \frac{F}{F_{0}}-3 \Omega_{\mathrm{m}}(\bar{z})\right),
\end{aligned}
$$

where GIF is given by $\gamma=\gamma_{0}+\gamma_{a} \frac{\bar{z}}{(1+\bar{z})}$ and we express all observables in terms of frame-independent variable, redshift $\bar{z}=z$.

We summarize the frame-(in)dependent quantities in table 2. As shown, the redshift, $z$ is a frameindependent measurable quantity. Thus, if one express frame-dependent observables, the Hubble parameter, (luminosity distance, angular diameter distance) in terms of $z$, then one can compare measured quantities between two frames.

Now, we compare the observed quantities both in the JF and in the EF. We show the evolutions of Hubble parameters, $\bar{H}$ and $H_{\mathrm{E}}$ in both frames in Fig. 1 . We assume that both the background evolution 
Table 2. Frame-(in)dependence of (im)measurable quantities. In measurable column, $X$-marks mean unmeasurable and $\sqrt{ }$-marks denote measurable. In equality columns, $\boldsymbol{X}$-mark imply inequality and $\checkmark$-marks mean equality.

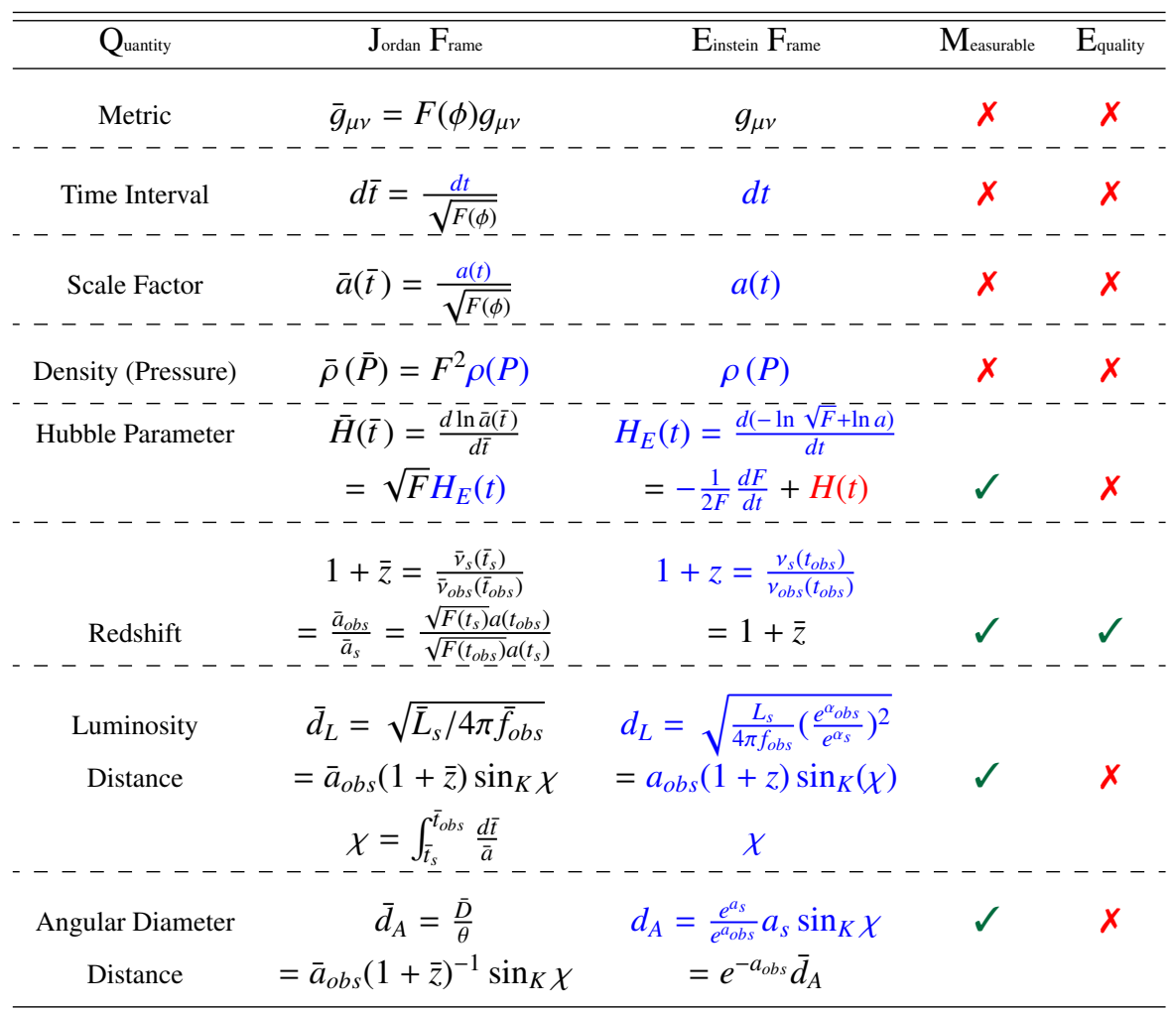

and the large scale structure formation provide $\omega=-1.1+0.2 \frac{z}{1+z}$ and $\gamma=0.6$ [7]. The solid line describes the $\bar{H} / \bar{H}_{0}$ in the JF and the dashed line depicts the $H_{\mathrm{E}} / H_{\mathrm{E} 0}$ in the EF. The difference of Hubble parameters between two frames is as large as $9 \%$ at present. We also show the evolutions of DE EOSs in both frames at Fig. 2. The solid line and the dashed line describe that of the JF and of the EF, respectively. The JF EOS is exactly same as the measured one, $\bar{\omega}=\omega=-1.1+0.2 \frac{z}{1+z}$ as expected. However, the EF EOS differs from that of the JF $0 \%(z=1.4) \leq \delta \omega \leq 30 \%(z=0)$. 


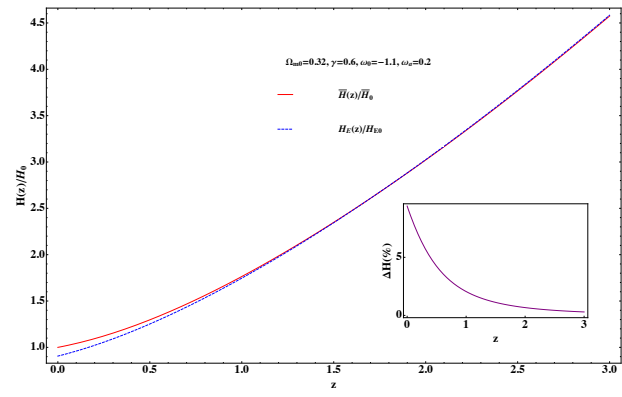

Figure 1. Evolutions of Hubble parameter both in the JF and in the EF. The solid line and the dashed line depict $\bar{H}^{2} / \bar{H}_{0}^{2}$ and $H_{\mathrm{E}}^{2} / H_{\mathrm{E} 0}^{2}$, respectively.

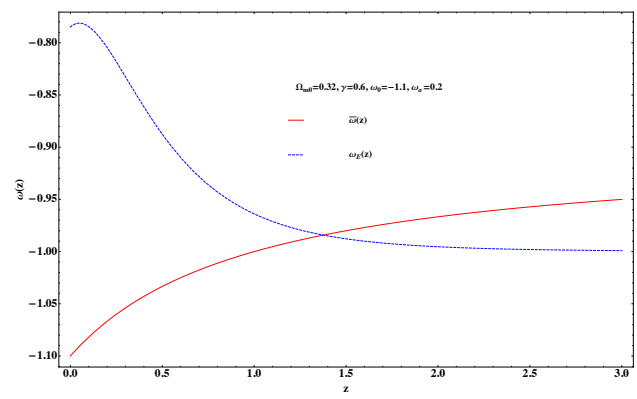

Figure 2. Evolutions of DE EOS both in the JF and in the EF. The solid line and the dashed line depict $\bar{\omega}$ and $\omega_{\mathrm{E}}$, respectively.

\section{Conclusions}

In this manuscript, we explicitly show how to reconstruct the model functions in scalar-tensor gravity theory from the observed parameters, $\omega$ and $\gamma$. Thus, if observational measurements on both parameters are very accurate, then the dynamics of model functions is determined. From this reconstruction, one can compare the Jordan frame cosmological observables like Hubble parameter and luminosity function with those in the Einstein frame. We explicitly show that the cosmological observables are frame-dependent under conformal transformation. Thus, one should choose a frame first before analysis of any observable and should keep using the same frame in the interpretation of other observable quantities. This demonstrates the explicit conformal inequality for cosmological observables.

\section{Acknowledgments}

SL and YK are supported by Basic Science Research Program through the National Research Foundation of Korea (NRF) funded by the Ministry of Science, ICT and Future Planning (Grant No. NRF2017R1A2B4011168 and NRF-2016R1D1A1B03931090, respectively).

\section{References}

[1] M. Chevallier and D. Polarski, Int. J. Mod. Phys. D 10, 213 (2001) [arXiv:gr-qc/0009008].

[2] E. V. Linder, Phys. Rev. Lett. 90, 091301 (2003) [arXiv:astro-ph/0208512].

[3] S. Lee and K.-W. Ng, Phys. Rev. D 82, 043004 (2010) [arXiv:0907.2108].

[4] G. Esposito-Farèse and D. Polarski, Phys. Rev. D 63, 063504 (2001) [arXiv:gr-qc/0009034].

[5] T. Chiba and M. Yamaguchi, J. Cosmol. Astropart. Phys. 1310, 040 (2013) [arXiv:1308.1142].

[6] S. Lee, J. Cosmol. Astropart. Phys. 1103, 021 (2011) [arXiv:1012.2646].

[7] S. Lee, [arXiv:1205.6304]. 